\title{
The Case for Streaming Multimedia with TCP
}

\author{
Charles Krasic, Kang Li, and Jonathan Walpole* \\ Oregon Graduate Institute, Beaverton OR 97206, USA, \\ $\{$ krasic, kangli, walpole\}@cse.ogi.edu, \\ WWW home page: http://www.cse.ogi.edu/sysl/
}

\begin{abstract}
In this paper, we revisit and challenge the dogma that TCP is an undesirable choice for streaming multimedia, video in particular. For some time, the common view held that neither TCP nor UDP, the Internet's main transport protocols, are adequate for video applications. UDP's service model doesn't provide enough support to the application while TCP's provides too much. Consequently, numerous research works proposed new transport protocols with alternate service-models as more suitable for video. For example, such service models might provide higher reliability than UDP but not the full-reliability of TCP. More recently, study of Internet dynamics has shown that TCP's stature as the predominant protocol persists. Through some combination of accident and design, TCP's congestion avoidance mechanism seems essential to the Internet's scalability and stability. Research on modeling TCP dynamics in order to effectively define the notion of TCP-friendly congestion avoidance is very active. Meanwhile, proposals for video-oriented transport protocols continue to appear, but they now generally include TCPfriendly congestion avoidance. Our concern is over the marginal benefit of changing TCP's service model, given the presence of congestion avoidance. As a position paper, our contribution will not be in the form of final answers, but our hope is to convince the reader of the merit in reexamining the question: do applications need a replacement for TCP in order to do streaming video?
\end{abstract}

\section{Introduction}

The Internet's ubiquity has long made it an attractive platform for distributed multimedia applications. A particularly elusive goal has been effective streaming solutions. To prevent confusion, we clarify the distinction between streaming and other forms of distribution, namely download. We assume download is defined so that the transfer of the video must complete before the video is viewed. Transfer and viewing are temporally sequential. With this definition, it is a simple matter to employ quality-adaptive video. One algorithm would be to deliver the entire video in the order from low to high quality components. The user may terminate

\footnotetext{
* This work was partially supported by DARPA/ITO under the Information Technology Expeditions, Ubiquitous Computing, Quorum, and PCES programs and by Intel
} 
the download early, and the incomplete video will automatically have as high quality as was possible. Thus, quality-adaptive download can be implemented in an entirely best-effort, time-insensitive, fashion. On the other hand, we assume streaming means that the user views the video at the same time that the transfer occurs. Transfer and viewing are concurrent. There are timeliness requirements inherent in this definition, which can only be reconciled with best-effort delivery through a time-sensitive adaptive approach.

In considering TCP's viability for streaming video, our position has much in common with the recent proliferation of work on TCP-friendly streaming. For us, the important issue is whether TCP's service model need to change. Much of the TCP-friendly research does not involve changes to the programming interface, our position is concerned with proposals that do entail new service models.

\section{Anti-TCP Dogma}

Numerous works on streaming video have asserted that TCP is undesirable for multimedia streaming, yet propose alternate solutions compatible with the same best-effort IP infrastructure $[3,9,16,15]$. In this section, we identify common objections to two of TCP's basic mechanisms, packet retransmissions and congestion avoidance, that are at the root of this anti-TCP dogma.

\subsection{Reliability through retransmissions}

One objection states that TCP's use of packet retransmissions introduces unacceptable end-to-end latency. The claim is that re-sending lost data is not appropriate because, given the real-time nature of video, the resent data would arrive at the receiver too late for display. This latency constraint can be addressed through client-side buffer management - if it does not conflict with applicationlevel latency requirements. With TCP, the point of conflict will occur when the latency requirements of the application are close to path round-trip time (RTT). A TCP sender's earliest detection of lost packets occurs in response to duplicate ACKs from the receiver, therefore the earliest time the re-transmission will arrive at the receiver is one full round-trip after the original data was lost ${ }^{1}$.

For purely-interactive applications such as tele-conferencing or distributed gaming, users are highly sensitive to end-to-end delays of sub-second timescales, typically in the range of 150 to 200 milliseconds. This end-to-end delay requirement persists for the duration of these applications. Unlike purely-interactive applications, video on demand (VOD) has interactive requirements only for control events such as start, pause, fast-forward, etc., which are relatively infrequent compared to the normal streaming state. While streaming, the quality perceived by the user is not directly affected by end-to-end latency, as the interaction

\footnotetext{
1 There is no bound on TCP's contribution to end-to-end latency, since the underlying IP model implies that acknowledgments or packet retransmissions may be lost. However, retransmission-delay on the order of a single RTT is the normal case.
} 
is strictly uni-directional. A VOD application may gradually increase buffering, hence end-to-end delay, by dividing its use of available bandwidth between servicing video play-out and buffer accumulation. After a time, the end-to-end delay will actually be quite large, but the user perceives it only indirectly, in the sense that quality during the buffer accumulation period might have been slightly decreased. In this way, we say that VOD does not have the inherent hard latency requirements of purely-interactive applications, and so TCP's packetretransmissions are not a significant problem for VOD.

For purely-interactive applications, we think it is important to characterize delays using the critical-path approach $[11,2]$. The question is how much of the retransmission-delay effects mean and worst-case critical-paths for interactive applications. The critical path approach stresses the importance of interaction with other sources of delay. If congestion avoidance is essential to the best-effort Internet, it may be that its delays dominate the critical path. Our experience in implementing a streaming VOD system has been that TCP's reliable service model greatly simplifies the application[8]. For these reasons and more to come in the following sections, we think it is entirely an open question whether TCP's retransmissions are appropriate for Internet video applications, even in the purely-interactive case.

\subsection{Congestion Avoidance}

The congestion avoidance algorithms of TCP have been heavily studied and frequently discussed in the literature[4,6,13]. Briefly, the congestion algorithm is designed to probe available bandwidth, through deliberate manipulation of the transmission rate. In steady-state, TCP's congestion control converges on an average transmission rate close to a fair-share of available bandwidt $h^{2}$. When viewed over shorter time-scales, TCP's instantaneous transmission rate takes on a familiar sawtooth shape, where it cycles between periods of additive increase separated by multiplicative decrease (AIMD). This short-term rate sawtooth is the second major part of the common view that TCP is not a good selection for video applications.

Many TCP-friendly protocols with claims of better suitability for video have been proposed[3, 9, 16, 15, 17]. These protocols recognize the need for congestion avoidance, but propose congestion avoidance such that rate is smoother in the short-term than TCP's AIMD sawtooth. Discussion in the literature of the network implications in terms of efficiency, stability and scalability, continues. We now consider the implications from the perspective of a streaming video application, which are manifest in terms of relationship between rate variations and buffering.

An application's TCP flow experiences rate variations for two distinct reasons; the first being competing traffic in the network, and the second being the

\footnotetext{
${ }^{2}$ Fairness under distributed control is necessarily somewhat subjective. TCP's control algorithm results in bias toward flows with shorter path RTTs.
} 
flow's own congestion avoidance behavior ${ }^{3}$. Rate variations may be categorized by the application as either transient or persistent. The distinction between transient and persistent rate changes is whether the buffer capacity is large enough to smooth them out. The purpose of buffering is precisely to smooth out transient changes.

For any amount of buffering, competing traffic can have persistent effects on a stream's rate. Streaming video applications must deal with persistent rate changes, before the client-side buffers are overwhelmed. The usual way is to employ quality-adaptation, adjusting the basic quality-rate trade-off of the video[3, $9,16]$. The applications use a closed loop-feedback control between client and server, which monitors the transport's progress for persistent rate changes and actuates the stream's quality-rate trade-off in response. We call this the qualityadaptation control.

Conceptually, the cyclic nature of congestion avoidance's increase-decrease phases suggests it should be treated strictly as a source of transient rate changes. TCP's service model does not provide the application any explicit information to distinguish rate variations induced by changes in traffic conditions from the normal rate adjustments of the flow's own congestion avoidance behavior. If the quality-adaptation control is intended only to adjust for persistent traffic changes, then it has the problem of masking out the TCP sawtooth by inference. Without direct information, the quality-adaptation control may be less than optimal in terms of responsiveness. From the perspective of the human viewer, frequent video-quality changes are annoying, hence quality-adaptation controls are likely to biased toward stability through the use of damping. This damping will necessitate the use of larger buffers, through techniques described in section 2.1 , in effect expanding the scope of rate changes considered transient. Thus once again, congestion avoidance is not a significant problem for VOD applications.

On the other hand, for purely-interactive applications, it may not be possible to treat congestion-avoidance adjustments as transient, since end-to-end latency and buffer capacity are constrained. In this case, the design of the qualityadaptation control will have to choose between having higher average quality, allowing quality to track the sawtooth, or smoother quality, by forgoing available bandwidth during part or all of the peak bandwidth periods. Relative to applications like VOD, on the same path, a purely-interactive video application must accept some combination of more-variable or lower-average quality. New congestion controls may reduce the magnitude of these penalties, since they may spread the congestion avoidance rate adjustments more evenly[15, 5].

\section{Popularity and Momentum}

Studies of traffic trends in the Internet suggest that applications based on TCP comprise most of the traffic[12]. Solutions that allow Infrastructure providers to improve network efficiency and application performance without changing the

\footnotetext{
3 TCP's flow control may also contribute, but for our discussion we assume the client
} is not the limit. 
applications are naturally compelling, so there is a strong incentive to improve TCP. A proposal that delivers better service to existing customers has more credibility than one that must attract new customers before it can pay-off. At the moment, video comprises a small minority of Internet usage, so video-only oriented transports have limited immediate appeal. Also, video-only transport proposals must struggle to overcome resistance based on their potential to disrupt existing majority of TCP based traffic. Meanwhile, improvements for TCP will move the performance target. We give two examples: Early Congestion Notification (ECN) and ATCP.

TCP's congestion control was predicated on the assumption that router buffer overflows were by far the most common source of packet losses. Accordingly, TCP's congestion avoidance mechanism relies on packet losses to support probing for bandwidth and congestion detection, which implies a certain amount of deliberate waste. ECN is a proposal for extending IP and TCP so that active queue management at network nodes can pro-actively inform TCP of congestion before packet losses occur[14]. While the retransmission mechanism is still necessary for TCP's reliable service model, ECN allows TCP to perform congestion avoidance without packet losses. Performance evaluation of ECN shows that ECN-enabled TCP connections usually proceed with little or no retransmissions over their lifetime[1]. While this has immediate implications for interactive video, it also leads to solutions of another deficiency in TCP, namely its performance over the expanding component of the Internet consisting of wireless ad hoc networks. It is well known that Wireless links often suffer high bit error rates, which standard TCP will mis-interpret as congestion. Invoking congestion avoidance for such errors impacts throughput more than is necessary, and is basically the wrong response. Liu and Singh[10] show that with ECN, it is possible to distinguish physical link losses from buffer overflows (congestion), and preserve TCP's throughput. While ECN and ATCP face deployment issues, the scope of change they propose is relatively modest, and yet they deliver comparable benefits to new protocols with video-centric service models.

\section{Discussion}

Our intuition is that interface preserving improvements to TCP are likely to benefit video streaming in proportion to their benefit for traditional applications. For new transport interfaces, we should expect a complete picture of their advantages and disadvantages. Any such picture is incomplete if it doesn't include a diligent comparison to a TCP based solution. Furthermore, the comparison must be include realistic measures of video quality impacts, rather than just network level measures. The motivation of stating our position is to set the context for a future presentation of our work on quality-adaptive video streaming. We have implemented a quality-adaptive video system that supports tailorable finegrained adaptation of MPEG derived video through priority packet dropping[7]. Due to space constraints, we omit preliminary description of our application's algorithm for streaming over TCP, they are available in the expanded technical 
report[8]. Our current work plans are to measure and quantify the performance of our streaming system, in terms that relate application-level (video) quality measures to transport throughput and to buffering and latency constraints. We expect to show our quality-adaptation exposes good controls over transient and persistent rate variations, without modifications to TCP .

\section{References}

1. Uvaiz Ahmed and Jamai Hadi Salim. Performance evaluation of explicit congestion notification (ECN) in IP networks. IETF RFC 2884, July 2000.

2. Paul Barford and Mark Crovella. Critical Path Analysis of TCP Transactions. In In Proceedings of the 2000 ACM SIGCOMM Conference, September 2000.

3. Shanwei Cen and Jonathan Walpole. Flow and congestion control for internet streaming applications. In Proceedings Multimedia Computing and Networking (MMCN98), 1998.

4. Dah-Ming Chiu and Raj Jain. Analysis of the Increase and Decrease Algorithms for Congestion Avoidance in Computer Networks. Computer Networks and ISDN Systems, 17, 1989.

5. Nick Feamster, Deepak Bansal, and Hari Balakrishnan. On the Interactions Between Layered Quality Adaptation and Congestion Control for Streaming Video. In 11th International Packet Video Workshop (PV2001), Kyongiu, Korea, April 2001.

6. Van Jacobson and Michael J. Karels. Congestion Avoidance and Control. In In Proceedings of ACM SIGCOMM'88, pages pp. 79-88, August 1988.

7. Charles Kasic and Jonathon Walpole. Qos scalability for streamed media delivery. CSE Technical Report CSE-99-011, Oregon Graduate Institute, September 1999.

8. Charles Krasic, Jonathan Walpole, Kang Li, and Asvin Goel. The case for streaming multimedia with tcp. Technical report, Oregon Graduate Institute, CSE Technical Report 2001. CSE-01-003.

9. J.R. Li, D. Dwyer, and V. Bharghavan. A transport protocol for heterogeneous packet flows. In IEEE Infocom'99, 1999.

10. J. Liu and S. Singh. ATCP: TCP for Mobile Ad Hoc Networks, 2001.

11. K. G. Lockyer. Introduction to Critical Path Analysis. Pitman Publishing Co., New York, N.Y., 1964.

12. S. McCreary and K. Claffy. Trends in Wide Area IP Traffic Patterns: A View from Ames Internet Exchange.

13. Jitendra Padhye, Victor Firoiu, Don Towsley, and Jim Kurose. Modeling TCP Throughput: A Simple Model and its Empirical Validation. In In Proceedings of ACM SICOMM'98, 1998.

14. K. K. Ramakrishnan, Sally Floyd, and D. Black. The Addition of Explicit Congestion Notification (ECN) to IP. IETF Internet-Draft, January 2001.

15. R. Rejaie, M. Handley, and D. Estrin. RAP: An end-to-end rate-based congestiong control mechanism for realtime streams in the internet. In Proceeedings of IEEE Infocomm, March 1999.

16. Wai tan Tan and Avideh Zakhor. Internet video using error resilient scalable compression and cooperative transport prototocl. In Proc. ICIP, volume 1, pages 17-20, 1998.

17. The TCP-friendly website. http://www.psc.edu/networking/tcp_friendly.html. 